\title{
Is Gd-DTPA Necessary in Clinical Practice for Peripheral Nerve Injury?
}

W e read with interest the article published in the American Journal of Neuroradiology by Hill et $\mathrm{al}^{1}$ concerning the enhancement characteristics at $4.7 \mathrm{~T}$ of the sciatic nerve of rats after injury. The purpose of this article was to provide a reliable highresolution diagnostic method to judge the severity of trauma; in the clinical setting, this would guide future microsurgery repair. Sequences realized were axial T1-weighted gradient-echo with fat saturation before and after $0.1 \mathrm{mmol} / \mathrm{kg}$ of intravenous Gd-DTPA and axial T2-weighted rapid acquisition relaxation excitement with fat saturation, in addition to dynamic contrast-enhanced T1-weighted gradient-echo imaging with fat saturation and spectroscopy sequences.

Finding a reliable noninvasive diagnostic tool to determine the severity and injury of a nerve has a real impact in daily clinical practice because this type of lesion is very disabling and generally occurs in younger patients. An increase in uptake is expected after 7 days as mentioned by the authors because that is when the blood-nerve barrier permeability increases.

In our opinion, it would be interesting if the authors had compared the abnormalities visible with the contrast agent with the T2-weighted rapid acquisition relaxation excitement with fat saturation, for example, size, tumefaction, inhomogeneity of signal, and so forth, as well as validated it with histology first to create models of the type and severity of lesions. In the end, they could have recognized, for example, whether the most inhomogeneous lesions are those that more readily regenerate. Second, it would have been useful to establish whether the same findings were present in both sequences. Due to accumulation of the paramagnetic

http://dx.doi.org/10.3174/ajnr.A5637 contrast agent in the basal ganglion, ${ }^{2,3}$ as recently demonstrated, there has been a tendency to use fewer paramagnetic contrast agents, so the implementation of these results in clinical practice would be difficult.

Finally, ultra-high fields are beginning to be used with volunteers and patients for the analysis of peripheral nerves. We are currently in the validation and learning phase and now know that it is possible to delineate not only the nerve itself but also almost microscopic structures. We believe this finding will also allow us to classify the grade of injury and help determine which lesions have a better prognostic value.

\section{REFERENCES}

1. Hill BJ, Padgett KR, Kalra V, et al. Gadolinium DTPA enhancement characteristics of the rat sciatic nerve after crush injury at $4.7 \mathrm{~T}$. AJNR Am J Neuroradiol 2018;39:177-83 CrossRef Medline

2. Kanda $T$, Ishii $K$, Kawaguchi $H$, et al. High signal intensity in the dentate nucleus and globus pallidus on unenhanced T1-weighted MR images: relationship with increasing cumulative dose of a gadolinium-based contrast material. Radiology 2014;270:834-41 CrossRef Medline

3. Ramalho J, Castillo M, AlObaidy $\mathrm{M}$, et al. High signal intensity in globus pallidus and dentate nucleus on unenhanced T1-weighted MR images: evaluation of two linear gadolinium-based contrast agents. Radiology 2015;276:836-44 CrossRef Medline

(D) M.I. Vargas

Division of Neuroradiology Department of Imaging and Medical Information Sciences Geneva University Hospital Geneva, Switzerland (1) O. Ipek Ecole Polytechnique Federale de Lausanne Center for Biomedical Imaging Lausanne, Switzerland 